\title{
External Carotid Artery Stenting and Superficial Temporal Artery to Middle Cerebral Artery Anastomosis for Internal Carotid Artery Occlusion With External Carotid Artery Severe Stenosis
}

\author{
-Case Report-
}

\author{
Takayuki OKU, ${ }^{1}$ Kenichiro NOGAMI, ${ }^{1}$ Hiroyasu KOIZUMI, ${ }^{1}$ Hideyuki IsHIHARA, ${ }^{1}$ \\ Shoichi KATO, ${ }^{1}$ Hirosuke FUJISAWA, ${ }^{1}$ and Michiyasu SUZUKI ${ }^{1}$
}

${ }^{1}$ Department of Neurosurgery, Yamaguchi University School of Medicine, Ube, Yamaguchi

\begin{abstract}
Superficial temporal artery (STA) to middle cerebral artery (MCA) anastomosis may have inadequate effects in patients with internal carotid artery (ICA) occlusion and severe stenosis of the ipsilateral external carotid artery (ECA), because poor blood flow in the STA leads to insufficient flow to the MCA. In these patients, dilation of the stenotic ECA is required to improve the blood flow in the STA before STAMCA anastomosis. A 71-year-old man presented with left hemiparesis and dysarthria. Magnetic resonance imaging revealed an old watershed infarction in the right cerebral hemisphere. Right carotid angiography showed right ICA occlusion and severe ipsilateral ECA stenosis. Single photon emission computed tomography (SPECT) demonstrated severe hemodynamic insufficiency in the right MCA territory. Instead of endarterectomy of the ECA, angioplasty and stenting (CAS) for ECA was performed to ensure adequate blood flow in the STA, due to the history of myocardial infarction and bifurcation of the common carotid artery at a high level (C2 level). Then STA-MCA anastomosis was performed 1 month later. Postoperative SPECT demonstrated marked improvement of hemodynamic insufficiency in the right MCA territory. After treatment, the patient had no ischemic events. This case suggests external CAS together with STA-MCA anastomosis is a good therapeutic option for a patient with symptomatic ICA occlusion and severe stenosis of the ipsilateral ECA if external CEA is difficult to perform.
\end{abstract}

Key words: carotid artery stenting, external carotid artery stenosis, hemodynamic insufficiency, internal carotid artery occlusion, superficial temporal artery-middle cerebral artery anastomosis

\section{Introduction}

Extracranial to intracranial (EC-IC) bypass surgery was reported to be ineffective for preventing further cerebral ischemia in patients with atherosclerotic internal carotid artery (ICA) or middle cerebral artery (MCA) diseases in 1985.3) However, further studies showed that this approach is effective in selected patients, especially in those with severe hemodynamic insufficiency. ${ }^{5,8,9,12,15)}$ The Japanese EC-IC Bypass Trial (JET Study) demonstrated the efficacy of superficial temporal artery (STA) to MCA anastomosis (STA-MCA anastomosis) for preventing new stroke in occlusive ICA and MCA diseases. ${ }^{9)}$ However, the effect of STA-MCA anastomosis may be inadequate because poor blood flow in the STA leads to insufficient flow to the MCA in patients with ICA occlusion and coexisting severe stenosis of the ipsilateral external carotid artery (ECA). In such patients, dilation of the stenotic ECA is

Received November 24, 2011;

Accepted March 5, 2012 necessary to improve blood flow in the STA before STAMCA anastomosis. Endarterectomy of the ECA has been performed for this purpose,,$^{6,7,11,13,14,20)}$ but endovascular surgery for ECA stenosis is uncommon. ${ }^{1,4,18,19)}$ Here, we report a case of symptomatic ICA occlusion with severe stenosis of the ipsilateral ECA, in which cerebral perfusion was improved by treatment with carotid artery angioplasty and stenting (CAS), and STA-MCA anastomosis.

\section{Case Report}

A 71-year-old man came to our hospital emergency room because of left hemiparesis and dysarthria. His medical history included hypertension, insulin-independent diabetes mellitus, and myocardial infarction. He had undergone CAS for cerebral infarction of the left hemisphere due to left ICA stenosis, and was taking warfarin (3 $\mathrm{mg} /$ day) and aspirin (100 mg/day). He had also undergone percutaneous coronary intervention for coronary artery stenosis. Magnetic resonance (MR) imaging demonstrated 


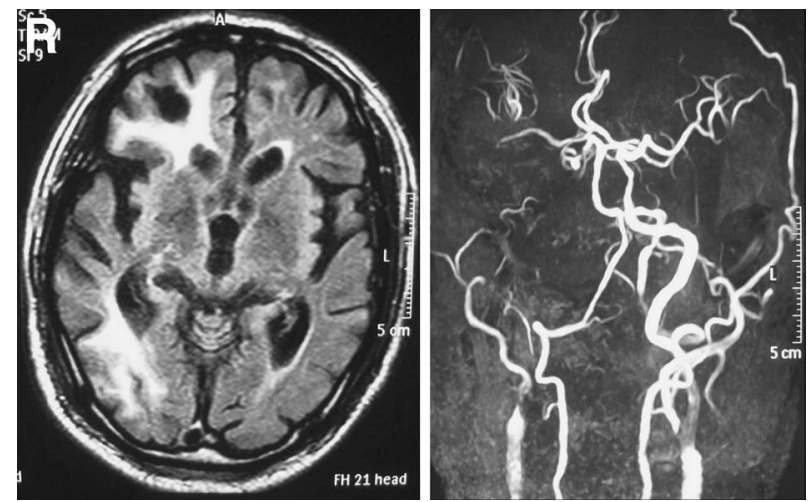

Fig. 1 Left: Fluid-attenuated inversion recovery magnetic resonance (MR) image showing an old watershed infarction in the right cerebral hemisphere. Right: MR angiogram showing occlusion of the right internal carotid artery and visualization of the right anterior cerebral artery and middle cerebral artery.

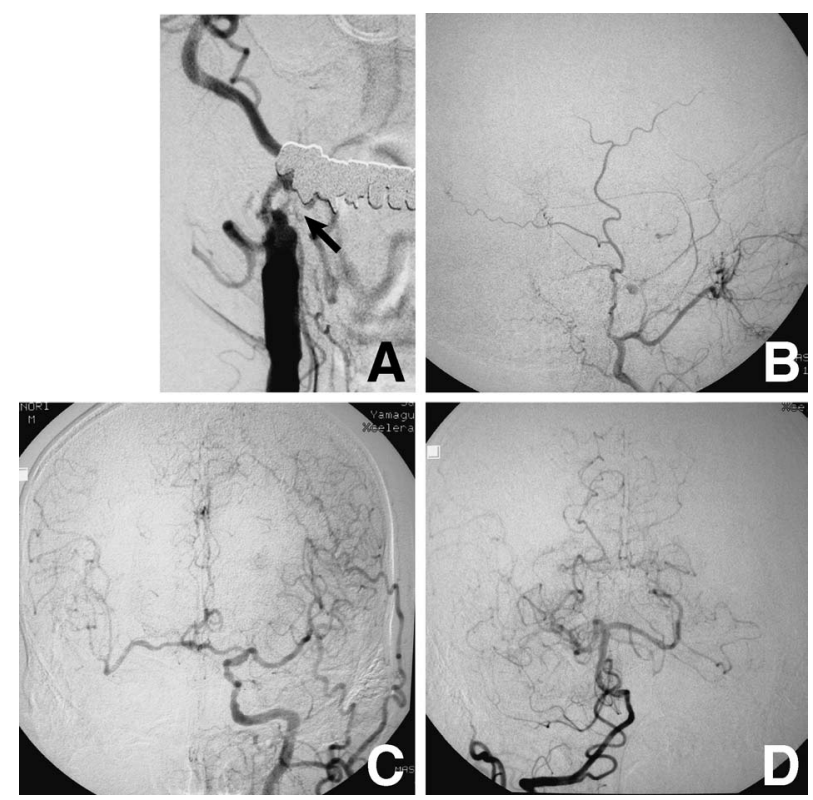

Fig. 2 A: Right carotid angiogram showing internal carotid artery occlusion and severe external carotid artery stenosis (arrow). B: Right carotid angiogram showing poor blood flow in the superficial temporal artery with little collateral blood flow via the ophthalmic artery. C: Left carotid angiogram showing the right anterior cerebral artery and middle cerebral artery via the anterior communicating artery. D: Right vertebral angiogram showing collateral blood flow via the posterior communicating artery and leptomeningeal anastomosis from the posterior cerebral artery.

only old watershed infarction in the right cerebral hemisphere (Fig. 1 left). Diffusion-weighted imaging revealed no fresh lesion. MR angiography showed occlusion of the right ICA (Fig. 1 right). He became asymptomatic rapidly after medical treatment, but transient left hemiparesis recurred frequently.

Carotid angiography showed right ICA occlusion and

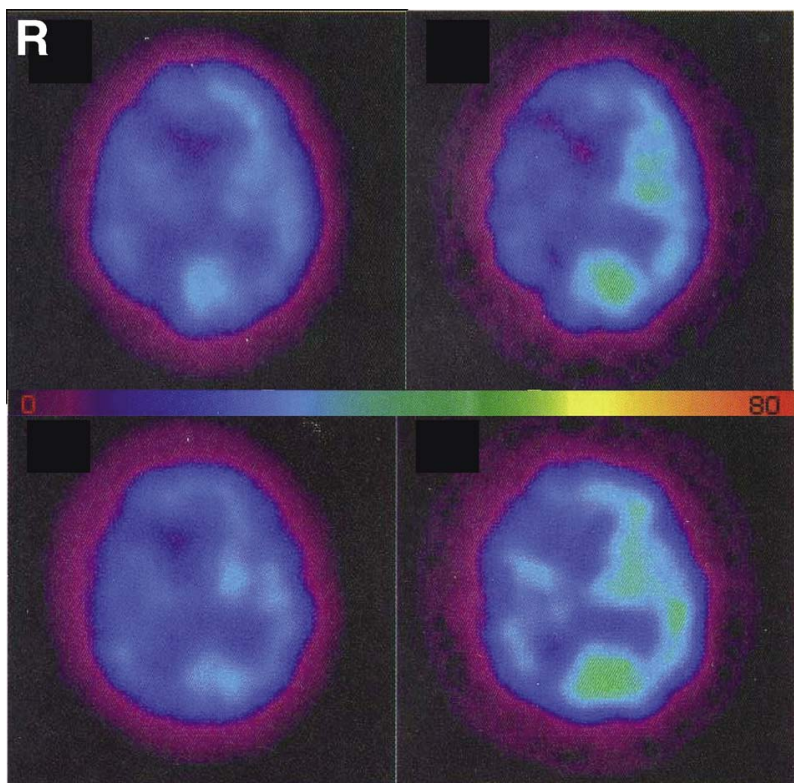

Fig. 3 Preoperative (upper row) and postoperative (lower row) single photon emission computed tomography (SPECT) scans with iodine-123 $\mathrm{N}$-isopropyl-p-iodoamphetamine at rest (left column) and after stress (right column). Preoperative SPECT scans demonstrating low resting cerebral blood flow (CBF) and markedly decreased regional cerebral vascular reserve (rCVR) in the right middle cerebral artery (MCA) territory, indicating hemodynamic insufficiency. Postoperative SPECT scans demonstrating low resting CBF and improved rCVR in the right MCA territory, indicating improvement of hemodynamic insufficiency. Color bar indicates the quantitative scale of $\mathrm{CBF}$ (ml/100 g/min).

severe stenosis of the ipsilateral ECA (Fig. 2A). The right STA appeared to have poor blood flow, and collateral flow via the ophthalmic artery was not apparent (Fig. 2B). Left carotid and right vertebral angiography demonstrated collateral blood flow via the anterior communicating artery (AcomA) and posterior communicating artery (PcomA) (Fig. 2C, D). Collateral flow from the posterior cerebral artery was also seen via a leptomeningeal anastomosis (Fig. 2D).

Single photon emission computed tomography (SPECT) with iodine-123 $\mathrm{N}$-isopropyl-p-iodoamphetamine was performed using the autoradiography method. Preoperative SPECT demonstrated low resting cerebral blood flow (CBF) in the right MCA territory (normal CBF is defined as $42.5 \mathrm{ml} / 100 \mathrm{~g} / \mathrm{min}$ in our institution). SPECT after injection of acetazolamide (Diamox) also demonstrated markedly decreased regional cerebral vascular reserve (rCVR), defined as (CBF with Diamox - resting CBF)/resting CBF $\times 100(\%)$, in the region of low CBF. Resting CBF, Diamox $\mathrm{CBF}$, and rCVR in the right MCA territory were 17.7 $\mathrm{ml} / 100 \mathrm{~g} / \mathrm{min}, 15.77 \mathrm{ml} / 100 \mathrm{~g} / \mathrm{min}$, and $-9.9 \%$, respectively (Fig. 3 upper row). These findings indicated that the patient had severe hemodynamic insufficiency and convinced us of the need for surgical treatment.

We planned to perform initial stenting for the ECA stenosis to ensure sufficient blood flow in the STA, with sub- 


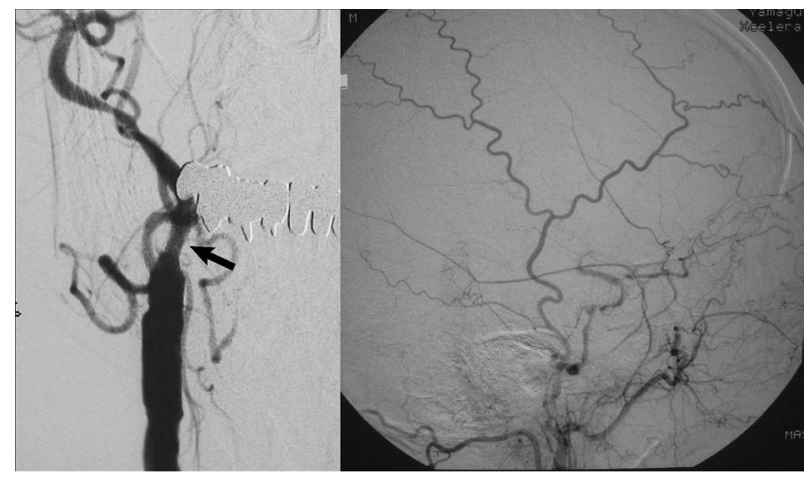

Fig. 4 Right carotid angiograms after stent placement showing marked reduction in stenosis (arrow), and improvement of flow in the superficial temporal artery and collateral circulation to the intracranial right internal carotid artery via the ophthalmic artery.

sequent STA-MCA anastomosis. Stenting was performed about 3 months after admission, due to complications of heart failure and intractable pneumonia. From 7 days before the procedure, he was given clopidogrel (75 mg/day) as well as continuing aspirin (100 mg/day). Warfarin was stopped. The activated clotting time was maintained over 300 seconds throughout the procedure by heparin intravenous injection. An $8 \mathrm{~F}$ guiding catheter was placed in the right common carotid artery approximately $6 \mathrm{~cm}$ proximal (C5 level) to the stenotic segment of the right ECA. Routine diagnostic angiography showed the diameter of the ECA was $4 \mathrm{~mm}$. An Angioguard XP (5.0-mm) (Cordis, Miami, Florida, USA) was positioned in the right ECA just proximal to the facial artery for distal protection. Over the Angioguard, a $3 \times 40-\mathrm{mm}$ balloon (Starling, Boston Scientific, Natick, Massachusetts, USA) was navigated into the right ECA and used for pre-dilation to 6 atm for $30 \mathrm{sec}$ onds. A $7 \times 30-\mathrm{mm}$ stent graft (Precise; Cordis) was positioned across the lesion and deployed carefully. Postdilation was performed with a $4 \times 20$-mm balloon (Amiia; Cordis) to $10 \mathrm{~atm}$ for 30 seconds. Bradycardia and decrease of blood pressure were not seen throughout the procedure. After post-dilation, right carotid angiography demonstrated marked reduction of ECA stenosis, and improvement of blood flow in the STA and collateral circulation to the intracranial right ICA via the right ophthalmic artery (Fig. 4). The patient suffered no ischemic attack during the 1 month after stenting to bypass surgery. One month after stenting, STA-MCA anastomosis was performed. The frontal and parietal branches of the STA were successfully anastomosed to supra- and infra-sylvian cortical branches of the MCA.

The postoperative course was uneventful. The patency of the STA-MCA anastomosis was confirmed by threedimensional computed tomography angiography (Fig. 5). Postoperative SPECT was performed 2 months after bypass surgery and demonstrated improvement of hemodynamic insufficiency. Resting CBF, Diamox CBF, and rCVR in the right MCA territory were $16.81 \mathrm{ml} / 100 \mathrm{~g} / \mathrm{min}$, $21.83 \mathrm{ml} / 100 \mathrm{~g} / \mathrm{min}$, and 30.9\%, respectively (Fig. 3 lower

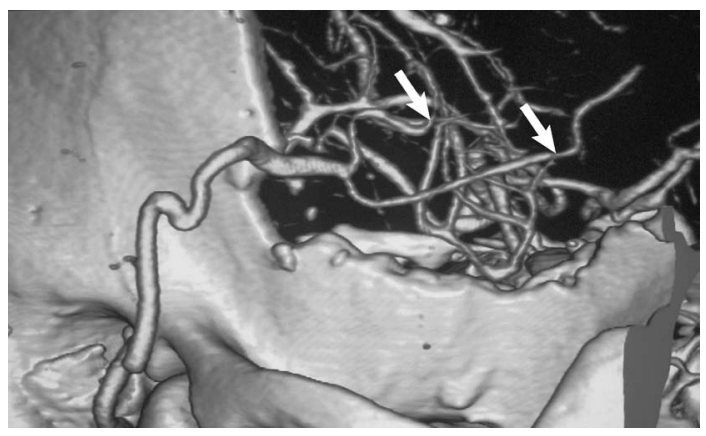

Fig. 5 Postoperative three-dimensional computed tomography angiogram showing patency of the superficial temporal artery to middle cerebral artery anastomosis. Arrows indicate the points of anastomosis.

row). Compared with preoperative SPECT, resting CBF was not significantly changed, but rCVR had markedly improved. Moreover, improvement of cerebral perfusion was also seen in the contralateral hemisphere. After treatment, the patient has taken clopidogrel (75 mg/day) and aspirin (100 mg/day), and has had no ischemic events for 11 months.

\section{Discussion}

Collateral blood flow from the ECA and via the AcomA and PcomA is very important in patients presenting with ICA occlusion. ${ }^{16,17)}$ Therefore, external carotid endarterectomy (CEA) is frequently performed for symptomatic patients with both ICA occlusion and severe ECA stenosis. ${ }^{6,7,11,13,14,20)}$ External CEA for progressive ECA stenosis improved hemodynamic insufficiency and symptoms in a patient who had undergone STA-MCA anastomosis for ipsilateral ICA occlusion. ${ }^{2)}$ Therefore, STA-MCA anastomosis can be chosen as a therapeutic option for patients in whom collateral flow from the ECA improves insufficiently and hemodynamic insufficiency remains even after external CEA. Recently, external CEA with STA-MCA anastomosis was effective for 7 patients with symptomatic ICA occlusion and advanced (>70\%) ECA stenosis. ${ }^{10)}$ On the other hand, reports of endovascular surgery for ECA stenosis are scarce. Good dilation of the ECA was obtained using percutaneous transluminal angioplasty for ECA stenosis, ${ }^{18)}$ and stenting was performed for ECA stenosis. ${ }^{1,4)}$ Recently, external CAS for patients with symptomatic ICA occlusion has been an effective strategy to preserve neurological function and to relieve ischemic symptoms with new development of compensated flow and/or increased rCVR. ${ }^{19)}$

In our patient, preoperative SPECT demonstrated low resting $\mathrm{CBF}$ and marked decrease of $\mathrm{rCVR}$ in the right MCA territory, and the patient suffered repeated transient ischemic attacks. Therefore, we concluded that he had severe hemodynamic insufficiency and needed aggressive reconstruction of cerebral perfusion. Endarterectomy seemed to have a higher risk of complication in our patient because of the history of myocardial infarction and bifur- 
cation of the common carotid artery at a high level (C2 level). For these reasons, we chose stenting over endarterectomy. After the operation, ECA stenosis was improved, and blood flow in the STA appeared to have increased sufficiently to supply adequate blood flow to the MCA after STA-MCA anastomosis. Postoperative SPECT demonstrated the combination therapy of external CAS and STA-MCA anastomosis had successfully increased rCVR (from $-9.9 \%$ to $30.9 \%$ ) and improved hemodynamic insufficiency in the right MCA territory. Therefore, this case suggests that such combination therapy is a good option for patients with symptomatic ICA occlusion and severe stenosis of the ipsilateral ECA if external CEA is difficult to perform.

\section{References}

1) Adel JG, Bendok BR, Hage ZA, Naidech AM, Miller JW, Batjer $\mathrm{HH}$ : External carotid artery angioplasty and stenting to augment cerebral perfusion in the setting of subacute symptomatic ipsilateral internal carotid artery occlusion. J Neurosurg 107: 1217-1222, 2007

2) Anegawa S, Shigekawa S, Hayashi T, Furukawa $Y$, Tomokiyo M, Torigoe R: Endarterectomy for narrowing of the external carotid artery in a patient who underwent extracranial-intracranial bypass for occlusion of the internal carotid artery—case report. Neurol Med Chir (Tokyo) 41: 442-445, 2001

3) The EC/IC Bypass Study Group: Failure of extracranial-intracranial arterial bypass to reduce the risk of ischemic stroke. Results of an international randomized trial. N Engl J Med 313: 1191-1200, 1985

4) Eisenberg JA, Dimuzio PJ, Carabasi A, Larson R, Lombardi JV: Endovascular repair of symptomatic external carotid artery stenosis. J Vasc Surg 42: 1210-1212, 2005

5) Garrett MC, Komotar RJ, Starke RM, Merkow MB, Otten ML, Sciacca RR, Connolly ES: The efficacy of direct extracranial-intracranial bypass in the treatment of symptomatic hemodynamic failure secondary to athero-occlusive disease: a system review. Clin Neurol Neurosurg 111: 319-326, 2009

6) Gertler JP, Cambria RP: The role of external carotid endarterectomy in the treatment of ipsilateral internal carotid occlusion: Collective review. J Vasc Surg 6: 158-167, 1987

7) Halstuk KS, Baker WH, Littooy FN: External carotid endarterectomy. J Vasc Surg 1: 398-402, 1984

8) Herzig $R$, Hlustík P, Urbánek $K$, Vaverka $M$, Burval $S$, Machác J, Vlachová L, Krupka B, Bártková A, Sanák D, Mares J, Kanovský P: Can we identify patients with carotid occlusion who would benefit from EC-IC bypass? Review. Biomed Pap Med Fac Univ Palacky Olomouc Czech Repub 148: 119-122, 2004

9) JET Study Group: [Japanese EC-IC Bypass Trial (JET Study): The second interim analysis]. Nosotchu No Geka 30: 434-437, 2002 (Japanese)

10) Kawamata T, Okada $Y$, Kawashima A, Yamaguchi K, Hori T: External carotid endarterectomy followed by superficial temporal artery to middle cerebral artery anastomosis for internal carotid artery occlusion with advanced ipsilateral external carotid stenosis. Neurosurgery 62: 395-399, 2008

11) Nicolosi A, Klinger D, Bandyk D, Towne J: External carotid endarterectomy in the treatment of symptomatic patients with internal carotid artery occlusion. Ann Vasc Surg 2: 336-339, 1988

12) Nussbaum ES, Erickson DL: Extracranial-intracranial bypass for ischemic cerebrovascular disease refractory to maximal medical therapy. Neurosurgery 46: 37-43, 2000

13) O'Hara PJ, Hertzer NR, Beven EG: External carotid revascularization: review of a ten-year experience. J Vasc Surg 2: 709-714, 1985

14) Satiani B, Das BM, Vasko JS: Reconstruction of the external carotid artery. Surg Gynecol Obstet 164: 105-110, 1987

15) Schaller B: Extracranial-intracranial bypass surgery to reduce the risk of haemodynamic stroke in cerebroocclusive atherosclerotic disease of the anterior cerebral circulationa systematic review. Neurol Neurochir Pol 41: 457-471, 2007

16) van Everdingen KJ, Visser GH, Klijin CJM, Kappelle LJ, van der Grond J: Role of collateral flow on cerebral hemodynamics in patients with unilateral internal carotid artery occlusion. Ann Neurol 44: 167-176, 1998

17) van Laar PJ, van der Grond J, Bremmer JP, Klijin CJ, Hendrikse J: Assessment of the contribution of the external carotid artery to brain perfusion in patients with internal carotid artery occlusion. Stroke 39: 3003-3008, 2008

18) Vitek JJ: Percutaneous transluminal angioplasty of the external carotid artery. AJNR Am J Neuroradiol 4: 796-799, 1983

19) Xu DS, Abruzzo TA, Albuquerque FC, Dabus G, Eskandari MK, Guterman LR, Hage ZA, Hurley MC, Hanel RA, Levy EI, Nichois CW, Ringer AJ, Batjer HH, Bendok BR: External carotid artery stenting to treat patients with symptomatic ipsilateral internal carotid artery occlusion: a multicenter case series. Neurosurgery 67: 314-321, 2010

20) Zarins CK, DelBeccaro EJ, Johns L, Turcotte JK, Dohrmann GJ: Increased cerebral blood flow after external carotid artery revascularization. Surgery 89: 730-734, 1980

Address reprint requests to: Takayuki Oku, MD, Department of Neurosurgery, Yamaguchi University School of Medicine, 1-1-1 Minami-kogushi, Ube, Yamaguchi 755-8505, Japan. 